Techniques \& Culture

\title{
La Formule de Mauss
}

Mauss'formula

\section{François Sigaut}

\section{(2) OpenEdition}

Journals

Édition électronique

URL : https://journals.openedition.org/tc/5007

DOI : $10.4000 /$ tc. 5007

ISSN : 1952-420X

\section{Éditeur}

Éditions de l'EHESS

\section{Édition imprimée}

Date de publication : 30 juin 2010

Pagination : 357-367

ISSN : 0248-6016

\section{Référence électronique}

François Sigaut, «La Formule de Mauss », Techniques \& Culture [En ligne], 54-55 | 2010, mis en ligne le 30 janvier 2013, consulté le 29 septembre 2022. URL : http://journals.openedition.org/tc/5007 ; DOI : https://doi.org/10.4000/tc.5007 


\section{LA FORMULE DE MAUSS}

in Techniques \& culture 40, 2002 : 153-168

Les ethnologues et les historiens s'interrogent évidemment fort souvent sur la validité des connaissances qu'ils produisent. Les expérimentations et les raisonnements logiques sont en principe reproductibles à volonté, à la seule condition d'en avoir le temps et les moyens matériels: c'est ce qui donne leur « dureté »-il vaudrait mieux dire leur solidité- aux sciences de la nature. Dans la plupart des sciences sociales au contraire, seuls les raisonnements sont aisément reproductibles, les faits le sont difficilement ou pas du tout. On pourrait de ce point de vue distinguer des faits singuliers, qui par définition n’arrivent qu'une fois et qui ne sont donc accessibles que par l'intermédiaire des témoignages ou des indices matériels qu'ils laissent, et des faits réguliers, qui bien que non reproductibles à volonté, se répètent assez souvent pour qu'on puisse les observer à loisir. L'intérêt pour les faits singuliers est à l'origine d'une abondante littérature de fiction, alors que les sciences sociales ont toujours marqué leur préférence pour les faits réguliers. Reste que la distinction entre les deux est assez artificielle. La statistique s'emploie depuis longtemps à faire apparaître des régularités dans les faits les plus singuliers. Quant aux faits réguliers, il est notoire que plus on les observe de près, plus ils tendent à s'individualiser et à devenir dissemblables les uns des autres. Si bien qu'en fin de compte, la qualification des faits de cette façon ne nous aide guère à résoudre le problème.

Une autre distinction possible est basée sur le caractère intentionnel ou non des faits dont on s'occupe. Il s'agit de la distinction philosophique classique entre causalité et intentionnalité, et même faudrait-il dire, intentionnalité consciente. Le domaine de la causalité est celui des faits matériels, soumis au déterminisme des lois de la nature, dont s'occupent les sciences proprement dites. Le domaine de l'intentionnalité est celui 
des choses de l'esprit, et il ne peut être objet de science dans la même acception du terme, parce que la science proprement dite suppose le déterminisme matériel, alors que l'esprit humain est censé se déterminer librement. On se souvient que c'est pour cette raison qu'Auguste Comte refusa de faire une place à la psychologie dans son système des sciences (quoiqu'il y acceptât la sociologie). D'autres parleront de «sciences de l'esprit », d'autres encore introduiront la distinction entre explication et interprétation. Expliquer un fait, c'est le rapporter à des causes matérielles comme cherchent à le faire les sciences de la matière. L'interpréter, c'est découvrir le sens qu'il a pour un sujet. Et lorsque les faits sont des actions humaines, c'est l'interprétation qui est pertinente, parce que c'est elle qui nous en donne la clef ${ }^{1}$.

Ces généralités sont aujourd'hui bien connues et je ne voudrais pas m'y attarder. Il me semble pourtant qu'elles ne sont pas sans implications sur le thème qui nous occupe. Parler d'interprétation suppose, bien sûr, qu'il y a quelque chose à interpréter, c'est-à-dire 1) que telle conduite d'autrui m'apparaisse comme ayant un sens mais 2) que ce sens ne me soit pas évident. Lorsque quelqu'un me parle dans une langue que j'ignore, je comprends qu'il me parle mais je ne comprends pas ce qu'il me dit, et c'est probablement cette combinaison spécifique de compréhension (d'une forme) et de noncompréhension (d'un contenu) qui crée le besoin d'interpréter.

Deux dernières remarques me paraissent utiles sur le couple explication/interprétation. La première est que l'interprétation n’a rien de spécifiquement scientifique. Dans la vie courante, nous passons notre temps à interpréter les faits et gestes de nos contemporains, ce qui est à la fois nécessaire socialement parlant et légitime sur un plan épistémologique - même si nombre de nos interprétations sont fausses, ce qui est une autre histoire. Mais nous passons aussi beaucoup de temps à interpréter des faits qui, eux, demanderaient plutôt à être expliqués. On pourrait parler d'animisme pour désigner cette tendance, qui n'est pas propre aux sociétés dites primitives, tant s'en faut. L'animisme correspond peut-être à quelque chose de spontané chez l'homme, bien que la conception d'une causalité spécifiquement mécanique le soit sans doute aussi. (Un des premiers à avoir proposé l'hypothèse d'une causalité directement perçue a sans doute été le psychologue A. Michotte vers 1950). Quoi qu'il en soit, il est généralement admis que les sciences de la nature se sont construites sur le rejet de l'animisme au profit de la causalité. Et comme les sciences sociales se sont elles-mêmes construites sur le modèle des sciences de la nature, on peut se demander si l'interprétation n'est pas un principe résiduel, dont le domaine d'application est voué à se réduire jusqu'à peut-être disparaître tout à fait.

Je ne suis pas de cette opinion, bien que je croie qu'il ne faille pas la sous-estimer. Ce qui est indéniable, c'est que dans la mesure où elle s'est voulue une discipline entièrement interprétative, l'anthropologie dite postmoderne (je me demande bien pourquoi) s'est enfermée dans une impasse. Car les activités humaines ne sont pas seulement intentionnelles, elles sont aussi et d'abord matérielles, le plus souvent même elles sont intentionnellement matérielles. Croire qu'on peut se contenter de les interpréter, c'est croire qu'on peut séparer l'intention de son objet, de ses moyens, de ses déterminations. C'est, en somme, réduire l'intentionnel à l'irrationnel, c'est-à-dire à l'arbitraire, au symbolique, pour ne pas dire au fantasme. Or, cette réduction est assez courante chez les ethnologues, et pas seulement chez ceux qui se rangent sous la bannière de l'anthropologie interprétative. Dans leurs conversations de couloir par exemple (c'est quelque chose qui m'a toujours étonné), les faits qui ne s'expliquent pas d'eux-mêmes sont souvent qualifiés de « culturels », 
comme s'il y avait le culturel d'un côté et le rationnel de l'autre - une opposition qui est d'ailleurs devenue d'actualité en sociologie des sciences. [...] C'est ainsi que dans le gros volume sur l'Ethnologie générale, paru dans La Pléiade en 1968, Jean Poirier écrit sans ambages que «l'univers du rationnel échappe à l'anthropologie » (p. 562).

Dans la perspective interprétative, chaque culture est une entité sui generis, avec ses concepts, ses valeurs et même sa rationalité propres, qu'on ne peut comparer à d'autres. Rien n'est vrai ou faux, juste ou injuste, logique ou illogique que selon les critères de telle ou telle culture. Et dans ces conditions, la seule validation possible est l'accord interne entre ceux qui s'en réclament. On aboutit à une sorte de solipsisme de groupe qui certes a toujours trouvé des adversaires et qui est aujourd'hui de plus en plus vigoureusement critiqué, mais qui n'en a pas moins gagné des positions dont l'importance se mesure, par exemple, à celle de l'idéologie dite du « politiquement correct ».

$[\ldots]$

Une autre idée à la mode aujourd'hui est que ce sont les controverses qui font avancer les sciences; je n'en suis pas tellement sûr. Tout dépend de ce qu'on entend par « controverse ». Il y a des pratiques de controverse qui stérilisent la pensée, comme celles qui s'étaient imposées dans l'université européenne à la fin du Moyen Âge. La science moderne n’a pu se développer, au début du xvII siècle, qu'après que ces pratiques, dites scolastiques, eurent été dûment critiquées et rejetées².

Les deux principales critiques qui ont été faites à l'anthropologie interprétative - exclusivement interprétative - ont été, me semble-t-il, d'autonomiser l'interprétation, c'est-à-dire de la séparer de l'explication, et de réifier la culture, c'est-à-dire d'en faire un facteur causal ou explicatif dans les conduites humaines (voir par exemple Keesing 1987). Ces critiques me semblent tout à fait pertinentes et nécessaires, mais je me demande si elles ne se situent pas à un niveau trop abstrait. Je ne crois pas, en effet, que les comportements des chercheurs obéissent à une logique aussi explicite. Et ce n'est pas avec des raisonnements qu'on expliquera le fait massif que les ethnologues, de quelque courant qu'ils se réclament, ne s'intéressent que marginalement aux techniques. En Afrique Noire, des millions de femmes passent plusieurs heures chaque jour à piler dans un mortier les grains ou les tubercules qui feront l'ordinaire quotidien de la famille. Or nous n’avons que très peu de descriptions ethnographiques détaillées de cette activité. On pourrait multiplier de tels exemples, qui vérifient fâcheusement le mot de Poirier. Plus les techniques sont « rationnelles », c'est-à-dire banales, familières, ordinairement efficaces, plus elles échappent à l'anthropologie. Il y a là un point qui mérite examen.

Dès 1903, Marcel Mauss, s’interrogeant sur les rites magiques, les définissait comme des " actes éminemment efficaces ", c'est-à-dire « capables de produire autre chose que des conventions; [...] ils sont créateurs; ils font ». Mais, ajoutait-il, « les techniques elles aussi sont créatrices. Les gestes qu'elles comportent sont également réputés efficaces. À ce point de vue, la plus grande partie de l'humanité peine à les distinguer des rites ». La suite du propos mérite d'être citée in extenso:

« La confusion [entre rites et techniques] est d'autant plus facile que le caractère traditionnel de la magie se retrouve dans les arts et dans les industries. La série des gestes de l'artisan est aussi uniformément réglée que la série des gestes du magicien. Cependant, les arts et la magie ont été partout distingués, parce qu'on sentait entre eux quelque insaisissable différence de méthode. Dans les techniques, l'effet est conçu comme produit mécaniquement. On sait qu'il résulte directement de la coordination des gestes, des 
engins et des agents physiques. On le voit suivre immédiatement la cause; les produits sont homogènes aux moyens: le jet fait partir le javelot et la cuisine se fait avec du feu. De plus, la tradition est sans cesse contrôlée par l'expérience qui met constamment à l'épreuve la valeur des croyances techniques. L'existence même des arts dépend de la perception continue de cette homogénéité des causes et des effets. » (Mauss 1950 [1902-1903] : 11-12).

Trente ans plus tard, Mauss ne fera que préciser un peu ces idées lorsqu'il proposera sa définition de la technique comme « acte traditionnel efficace... senti par l'auteur comme un acte d'ordre mécanique, physique ou physico-chimique, et poursuivi dans ce but » (Mauss 1950 [1934] : 371-372). Si aujourd'hui cette définition est assez bien connue, elle ne fait pas l'unanimité, même chez les ethnologues. Nul ne conteste que la technique soit acte, cela semble trop évident pour qu'il faille y insister. L'adjectif «traditionnel » suscite assez souvent des objections de la part de ceux qui opposent tradition et innovation, ou tradition et modernité. Mauss y avait répondu d'avance en disant qu'« il n'y a pas de technique et pas de transmission s'il n'y a pas de tradition ». On pourrait certes remplacer le mot par celui, plus courant aujourd'hui, de culture, et parler d'« acte culturel efficace »... Il me semble qu'on n'y gagnerait pas grand-chose. Dire qu'une technique ne peut exister que dans une tradition, c'est dire que pour exister, il faut qu'elle ait été apprise (ou inventée) et qu'elle soit pratiquée par quelqu'un, l'un et l'autre n'étant possibles qu'à l'intérieur de groupes sociaux qui ne sont pas n'importe lesquels, et qui ont donc leurs traditions propres. La morphologie des sociétés, c'est-à-dire la façon dont les humains s'agrègent en groupes et en sous-groupes identifiables et structurés, n'est pas seulement affaire de parenté, de pouvoir, de langage, de religion, etc. Elle est aussi affaire d'apprentissage et de pratique techniques. C'est un point que je crois tout à fait essentiel, bien que je ne puisse pas le développer ici.

Ce qui fait le plus de difficultés, c'est la notion d'efficacité. On n’a pas de mal à déceler un certain embarras dans les propos de Mauss sur ce point. Et dans un colloque récent, j'ai eu l'occasion d'entendre une critique tout à fait sérieuse du concept même de technique, portant essentiellement sur cette notion. Pour l'auteur de cette critique, toutes nos actions sont indissociablement symboliques et rituelles aussi bien que pratiques. Et lorsqu'une société croit bon de distinguer ces différents aspects les uns des autres, elle le fait à sa façon, qui n'est pas la même que celle de ses voisines et encore moins que la nôtre, Occidentaux d'aujourd'hui. En d'autres termes, ce qui est d'ordre purement pratique pour les uns sera un rituel hautement symbolique pour les autres, et ce qui est pour nous d'ordre religieux sera pour d'autres de l'ordre d'une physique des forces occultes, etc. Dans cette perspective, on ne peut pas comme le fait Mauss parler d'une efficacité « sentie par l'auteur comme d'ordre mécanique, physique ou physico-chimique » en dehors des sociétés de l'Occident moderne où il existe des sciences physiques - sauf à tomber dans l'ethnocentrisme.

Je crois que pour réfuter cette objection, il faut préciser la notion d'efficacité. Il faut en particulier la déconnecter de celle d'utilité. L'arbalète, le moulin à eau, la locomotive à vapeur, la moissonneuse-lieuse, etc., n'ont rien perdu de leur efficacité, mais elles n'ont plus d'utilité - sauf à titre d'attractions historico-touristiques. Être efficace, nous dit le dictionnaire, c'est " produire l'effet attendu », et ce n'est que cela. Il y a une manière efficace de faire des ricochets dans l'eau, puisque les débutants n'y réussissent pas du premier coup, mais les ricochets n'ont pas la moindre utilité, à moins de considérer comme utile l'amusement qu'on en retire. Précisons encore: l'effet attendu, c'est l'effet au sens exact que donnent les physiciens à ce terme quand ils parlent d'effet Joule, d'effet Doppler, 
etc. Ce qui signifie deux choses: 1) l'effet est sensible ou perceptible, que ce soit ou non par l'intermédiaire de certains appareils; et 2) sa perception est indépendante de toute croyance et de toute théorie. Des millions de gens de par le monde écoutent la radio et regardent la télévision sans avoir la moindre idée de la façon dont fonctionnent ces appareils (j'en fais moi-même partie). Aucun symbolisme, aucun système de pensée indigène n'y peuvent rien changer. Mais c'est exactement la même chose dans le maniement d'un outil, voire dans la marche à pied. Entre autres effets utilisés dans les deux cas, il y a le frottement: celui des mains sur le manche ou des pieds sur le sol. Le frottement est un phénomène physique fort complexe, que ceux qui ne sont pas physiciens ne comprennent certainement pas mieux que le fonctionnement d'un poste de télévision. Mais il n'y a nul besoin de le comprendre pour être capable de l'utiliser. Le bûcheron qui crache dans ses mains en perçoit immédiatement le résultat dans la tenue en main du manche de sa hache ou de sa serpe. Et l'expérience nous a appris de la même façon, parfois assez brutalement, à adapter notre démarche à la boue, à la neige, au verglas, voire aux parquets trop bien cirés. Si, comme le rappelle Marcel Mauss à juste titre, il y a différentes façons de marcher selon les sociétés, aucune d'entre elles ne dispense de regarder où on met les pieds. Pour que le concept de technique soit ethnocentré, comme le voudrait la critique que j'ai citée, il faudrait montrer qu'il existe des sociétés dans lesquelles même des activités aussi immédiatement physiques que cracher dans ses mains ou regarder où on met les pieds seraient liées à un symbolisme particulier ${ }^{3}$.

On pourrait parler d'effectivité pour éviter l'ambiguité d'efficacité. Mais à partir du moment où il est bien entendu qu'efficace renvoie à effet et non à utilité, je ne crois pas que cela soit indispensable. Il me semble plus intéressant de prolonger notre raisonnement en prenant la définition de Mauss à l'envers, pour ainsi dire. Si les techniques sont des actions traditionnelles efficaces, que seraient des actions non efficaces ou non traditionnelles? Il y a certes à un premier niveau des réponses évidentes: un échec, une action ratée, est évidemment inefficace. Mais il y a aussi une inefficacité volontaire, dans l'action simulée par exemple (théâtre, mime). Comment faire pour que les réponses évidentes ne nous cachent pas les autres, moins évidentes mais plus intéressantes peut-être? La méthode que j'ai suivie a consisté à partir des trois termes de la définition de Mauss, action, tradition et effet, et à en tirer un tableau dans lequel chacun des trois peut être marqué d'un (+) ou d'un (0) suivant qu'il est présent ou absent. Le résultat est le suivant:

\begin{tabular}{|c|c|c|l|}
\hline ACTION & TRADITION & EFFET & \\
\hline+ & + & + & Techniques ordinaires \\
\hline+ & + & 0 & $\begin{array}{l}\text { Échecs, pannes } \\
\text { Actions simulées (théâtre, mime...) }\end{array}$ \\
\hline+ & 0 & + & Techniques à effets paradoxaux \\
\hline 0 & + & + & Phénomènes naturels \\
\hline
\end{tabular}

Je me suis assez vite rendu compte, en réalité, que ce tableau n'était qu'une première esquisse, ouvrant peut-être quelques pistes à la réflexion mais peu satisfaisant en luimême. Si la première et la dernière ligne (techniques ordinaires, phénomènes naturels) ne semblaient pas offrir de difficultés, il n'en allait pas ainsi de la deuxième et de la troisième. L'échec et l'action simulée ne se situent pas sur le même plan, et n'épuisent d'ailleurs pas les possibilités d'action sans effet. Quant aux effets paradoxaux, ce sont des effets qui ne correspondent à rien de connu dans la culture du groupe considéré, qui 
contredisent même l'expérience commune de ses membres. Là encore, il y a au moins deux modèles, situés sur des plans différents: l'invention, quand elle produit des effets inédits mais réels (par exemple la plupart des applications de l'électricité à leurs débuts), et la prestidigitation qui produit des effets apparemment impossibles.

Après divers tâtonnements, il m'est apparu nécessaire de distinguer quatre plans ou quatre registres d'activité, qui sont: l'action proprement dite, ou utile, visant à l'obtention d'un effet « pour de vrai », si on me passe l'expression; l'apprentissage, où l'effet obtenu, s'il l'est, ne sert qu'à améliorer ou à vérifier l'habileté du sujet; le jeu, qui ressemble à l'apprentissage si ce n'est que les habiletés acquises ne sont pas destinées à servir « pour de vrai »; et enfin le spectacle, où il s'agit de montrer quelque chose à autrui. En tenant compte de ces quatre registres, on obtient un second tableau (ci-après), qui est en quelque sorte le développement du précédent.

Ce second tableau n'est pas encore tout à fait satisfaisant, il fait parfois un peu violence à la réalité. Mais il a l'avantage de donner une vision synoptique de tout un ensemble d'activités, qu'on peut trouver dans la plupart des sociétés même si ce n'est pas sous une forme aussi nettement institutionnalisée que les exemples présentés ici. Je ne vais pas commenter chacune de ces activités. Mon but, je le rappelle, n'est que de réfuter l'imputation d'ethnocentrisme qui pèse sur la conception maussienne de la technique. Il me semble que ce tableau y contribue, dans la mesure où il montre qu'en modifiant de façon ordonnée les composantes de l'action technique, on obtient d'autres sortes d'actions qui ont également un sens dans la plupart des sociétés.

La notion d'échec (case 5) par exemple n'a de sens que par rapport à l'action réussie correspondante (case 1), la différence étant que l'effet attendu n'est pas produit, ou incomplètement. Un faucheur qui s'arrête pour aiguiser sa faux le fait parce qu'il sent que celle-ci ne coupe plus comme il le voudrait: l'effet est bien identifié, même s'il n'est pas nécessairement conceptualisé. Avec les jeux de faire-semblant (7) et surtout le mime (8), l'effet est conceptualisé, puisqu'il est volontairement supprimé. Le but de l'action est alors de faire reconnaître ou deviner l'effet aux spectateurs, ce qui suppose que ceux-ci soient déjà familiarisés avec l'activité correspondante. C'est aussi vrai, quoique d'une façon différente, pour l'apprentissage par exercice (6) : l'action simulée, quelle qu'elle soit, n’a de sens que par référence à une action réellement efficace.

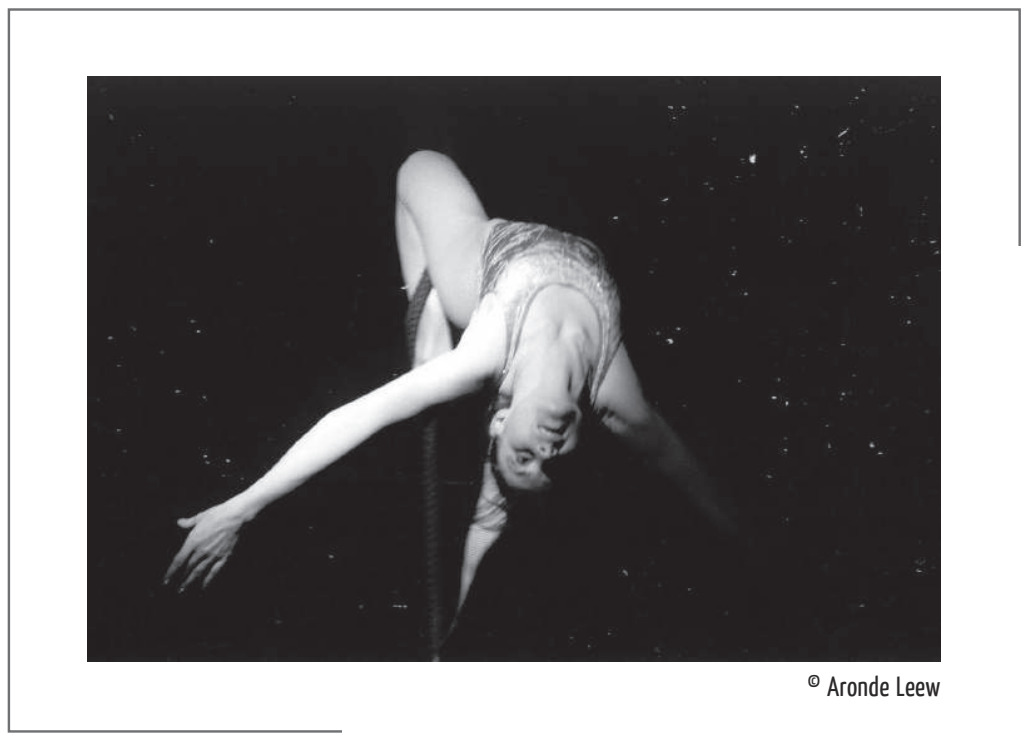


Les activités qui mettent en œuvre des effets que j’ai qualifiés de paradoxaux (cases 9 à 12) posent un problème un peu particulier, car un effet paradoxal ne peut pas le rester longtemps. Ou bien il n'est produit qu'une fois, et c'est un accident qui sera tôt ou tard oublié. Ou bien il est reproduit plus ou moins régulièrement, et il cesse d'être paradoxal.

Il faut reconnaître qu'à cet égard nous sommes aujourd'hui presque complètement blasés. On ne voit plus guère d'inventions susceptibles de créer d'aussi colossales sensations que les débuts de l'électricité, ceux de l'automobile, de l'aviation, de la radio, etc.

\begin{tabular}{|c|c|c|c|c|c|c|}
\hline ACTION & TRADITION & EFFET & FAIRE & APPRENDRE & JOUER & MONTRER \\
\hline+ & + & + & $\begin{array}{r}\text { Techniques « ordinaires » } \\
1\end{array}$ & $\begin{array}{l}\text { Entrainement par l'action } \\
\text { réelle (effective) }\end{array}$ & Jeux d’adresse & $\begin{array}{l}\text { Sports de compétition et de } \\
\text { démonstration ; acrobatie ; etc. } \\
\text { (cirque) } 4\end{array}$ \\
\hline+ & + & 0 & Échec, panne & $\begin{array}{l}\text { Entrainement par l'action } \\
\text { simulée (exercice) }\end{array}$ & Jeux de faire-semblant & Théâtre, mime. Certaines ruses, feintes \\
\hline+ & 0 & + & $\begin{array}{l}\text { (Premier essai d'une } \\
\text { invention?) }\end{array}$ & Expérimentation & Physique amusante & Prestidigitation, illusionnisme \\
\hline 0 & + & + & $\begin{array}{l}\text { Phénomènes spontanés } \\
\text { (indépendants de l'action } \\
\text { humaine) }\end{array}$ & (Révélation?) & Jeux de hasard & Automates \\
\hline
\end{tabular}

Pourtant, notre goût pour les effets paradoxaux reste vif, d'où sans doute le succès durable de la prestidigitation et de l'illusionnisme. Il s'agit certes d'effets dont le caractère paradoxal est simulé - nous savons pertinemment qu'il y a un truc - mais cela ne gâte pas notre plaisir. La prestidigitation est l'inverse du sport et de l'acrobatie, en quelque sorte. Le prestidigitateur met en scène des effets extraordinaires simulés, en cachant soigneusement les habiletés et les appareillages réels qui lui permettent de les produire. Le sportif et l'acrobate ne mettent en œuvre que des effets réels et ordinaires; c'est leur force, leur adresse, leur souplesse, etc., qui sont extraordinaires et mises en scène comme telles.

Y a-t-il des effets paradoxaux dans les sociétés pré-industrielles? Je n'ai guère d'informations à ce sujet, mais il est probable qu'on en trouverait, réels ou simulés, d'une part dans les jeux d'enfants (et d'adultes), d'autre part dans les pratiques des shamans, guérisseurs et autres magiciens. Le cerceau, la toupie, le cerf-volant, les ricochets dans l'eau, etc., mettent en œuvre des effets qui sont assurément connus, mais qui ont tout de même quelque chose de paradoxal dans la mesure où ils paraissent contrevenir à l'expérience la plus commune: le caillou rebondit sur l'eau, la toupie tient sur sa pointe... Depuis le xix ${ }^{e}$ siècle, les jeux de ce genre se sont multipliés, même si nombre d'entre eux ont été rapidement oubliés. Certains sont d'origine exotique, d'autres ressortissent à la mécanique amusante, d'autres enfin ont été inventés dans les milieux du sport et des jeux d'adresse. J'en sais trop peu sur cette histoire pour pouvoir en dire davantage. Mais chacun peut y réfléchir à partir des exemples qu'il connaît (le boomerang, le diabolo, le surf...) et en prêtant un peu d'attention aux jouets anciens ou curieux qu'il peut rencontrer ici et là.

La magie pose un problème plus difficile. Les effets magiques sont-ils ordinaires ou paradoxaux? À première vue, ils sont les deux à la fois: paradoxaux parce qu'impossibles à produire dans les circonstances ordinaires de la vie, mais ordinaires dans 
la mesure où la magie elle-même fait partie de l'expérience commune aux membres du groupe. À y regarder de plus près, toutefois, c'est l'aspect ordinaire qui tend à l'emporter. D'abord parce que le catalogue des effets imputés à la magie dans une société donnée, pour autant qu'on le connaisse, est toujours d'une insigne pauvreté - comme l'imagination humaine, quand elle se détache du réel. Ensuite parce que le recours à la magie, que ce soit pour attaquer ou pour se défendre, se fait toujours dans des conditions et suivant des formes prévues et codifiées par le groupe. Et enfin, parce que même dans les sociétés dites primitives, les effets paradoxaux proprement dits ne sont pas toujours ni nécessairement assimilés à des effets magiques. Dès leurs premiers contacts en Amérique et en Afrique Noire au Xvi siècle, et jusque dans les années 1940 en Nouvelle-Guinée, les Européens ont fait le plus large usage, pour impressionner les indigènes, des effets paradoxaux (pour ceux-ci) que leurs armes et leurs appareils leur permettaient de produire à bon compte. En l'absence d'études précises, nous n'avons guère que des clichés contradictoires sur la façon dont ces effets paradoxaux ont été interprétés par ceux auxquels ils étaient destinés. Rien, en tout cas, ne prouve qu'ils auraient été toujours et partout assimilés à des effets « magiques »-ce qui, d'ailleurs, impliquerait que la «magie » soit à peu près la même chose dans toutes les sociétés, hypothèse bien peu vraisemblable ${ }^{4}$.

Le plus inattendu dans notre tableau est sans doute l'existence de la dernière ligne (cases 13 à 16). Il pouvait paraître absurde de supprimer le premier terme de ce qu'on peut appeler la formule de Mauss, l'action elle-même. Or il s'est avéré possible de remplir les cases en question. Les phénomènes spontanés (13) n’appellent pas de commentaires. J'avais laissé vide la case 14, ce sont des étudiants qui m'ont fait remarquer qu'on pouvait y mettre les expériences de révélation. Dans la case 15 prennent place les jeux de hasard, non pas que l'action humaine en soit vraiment absente, mais parce que ce n'est pas elle qui est censée produire le résultat final: trop d'adresse dans ce genre de jeu suscite immédiatement la suspicion de tricherie. Il en est un peu de même avec les automates (16): l'action humaine n'est pas absente, mais elle est cachée ou déconnectée en apparence de l'action de la machine. Pendant des siècles, l'automate par excellence a été l'horloge, dont l'utilité sociale directe a toujours été bien moindre, me semble-t-il, qu'on ne l'a supposé. Depuis quelques lustres, on a vu apparaître un genre nouveau d'automates: les machines inutiles, considérées comme des œuvres d'art, où le jeu du mécanisme est mis en scène pour lui-même.

On a vu qu'avec ce tableau, ce que Mauss avait posé comme une définition était devenu une sorte de formule, la formule de la technique: action, tradition, effet. Nous avons vu également qu'il était possible d'annuler séparément chacun des trois membres de la formule, cette annulation étant réelle ou fictive. Il devient alors nécessaire de préciser le mieux possible les différents registres d'activités qui peuvent être concernés. J'en ai distingué quatre, peut-être y en aurait-il davantage. Mais mon propos n'est pas de dresser une sorte de tableau de Mendeleev des activités humaines. Il est de montrer que la formule de Mauss a une valeur analytique, et que cette valeur est transculturelle parce que toutes les sociétés humaines s'en servent elles-mêmes. Les apprentissages, les jeux, les spectacles peuvent être interprétés comme des formes pratiques d'analyse des techniques, qui ont un sens dans toutes les sociétés parce que la technique en a un. C'est parce qu'il y a une efficacité technique, reconnue comme telle, qu'il peut y avoir des apprentissages, des jeux d'adresse et de faire semblant, des actions mimées, des spectacles, etc. Dans Le rire (1993 [1899] : 51), Bergson observe: 
«S'il est vrai que le théâtre soit un grossissement et une simplification de la vie, la comédie pourra nous fournir, sur ce point particulier de notre sujet, plus d'instruction que la vie réelle. Peut-être même devrions-nous pousser la simplification plus loin encore, remonter à nos souvenirs les plus anciens, chercher, dans les jeux qui amusèrent l'enfant, la première ébauche des combinaisons qui font rire l'homme. »

Je me suis inspiré de cette idée de Bergson ici, non pour analyser le rire, mais pour montrer que l'homme ne fait pas que se servir de sa raison, il en joue. Opposer le rationnel et le culturel est une absurdité, résultat de l'application à l'ethnologie d'une conception philosophique classique mais étriquée, qui ne sait chercher la raison que dans le discours. Même dans cette perspective restreinte, d'ailleurs, on pourrait soutenir avec Descartes que chaque culture a sa part de bon sens universel, bien que ce ne soit pas l'argument le plus important. L'argument décisif, me semble-t-il, c'est qu'il existe un modèle universel du rationnel, qui est l'action traditionnelle efficace. Il s'agit d'un modèle universel, non seulement parce qu'il n'existe pas de groupe social sans techniques, c'est une évidence qui a souvent été rappelée, mais surtout, parce que toutes les sociétés inventent des jeux, des exercices ou des spectacles dans lesquels l'action traditionnelle efficace est analysée par la suppression, réelle ou fictive, de l'une de ses composantes. La formule de Mauss nous permet d'expliciter et de résumer la logique qui régit tout cet ensemble de conduites humaines. À nous d'en exploiter les possibilités. 


\section{NOTES}

1. Ces questions ont fait l'objet d'une abondante littérature philosophique au XIX ${ }^{e}$ siècle, principalement en Allemagne, dans laquelle des auteurs comme Dilthey (1833-1911) ou Windelband (1848-1915) font figure de chefs de file. Mais je n'ai pas l'impression que ce courant de réflexion ait produit des résultats concrets utilisables par les historiens ou les sociologues. En France en tout cas, les rares auteurs qui ont pris la peine d'en discuter se montrent plutôt sceptiques. Voir par exemple Henri Berr (1953: 24-25).

2. Dans les universités médiévales, la controverse était devenue un art en soi, pour ne pas dire un sport. Un sport qui avait ses champions, capables de « démontrer » en public n'importe quoi contre n'importe quel adversaire - ou qui du moins s'en vantaient. C'est lorsque la stérilité de ces joutes fut devenue manifeste que le recours à l'expérimentation put commencer à s'imposer comme une des bases de la pratique scientifique.

3. «En aucun temps, chez aucun peuple, il n'y eut un dieu de la pesanteur $»$ : par cette citation, dont il attribue la paternité à Auguste Comte après Adam Smith, Émile Meyerson (1931, I : 137-138) veut montrer que l'idée de loi naturelle a existé de tout temps, bien avant la science proprement dite, laquelle n'a fait que l'expliciter et en étendre systématiquement le champ d'application. Un autre auteur de la même époque, Louis Weber (1914 : 72) s'était servi de la même remarque pour établir que la notion de causalité est d'origine technique et non pas religieuse ou magique, comme le voulaient alors Durkheim et ses partisans. Très peu d'auteurs semblent s'être intéressés spécifiquement aux rapports actioneffet; parmi eux sont Henri Wallon (1941 : 48-58) et A. Michotte (1954: 19).

4. Explorateurs, militaires, missionnaires et même ethnologues ne se sont guère privés des facilités que leur donnait le matériel moderne (armes, machines, appareils de toutes sortes) pour impressionner les indigènes; les anecdotes sur ce sujet, si on pouvait les recenser, se compteraient par milliers. Le problème est que ces faits n'ont été rapportés que comme des anecdotes, alors qu'ils constituent un matériau de premier ordre pour comprendre ce qui se passe quand un de ces effets que j'ai appelés paradoxaux est produit dans un certain groupe social.

\section{RÉFÉRENCES}

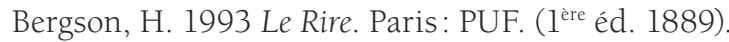

Berr, H. 1953 La Synthèse en histoire. Paris: Albin Michel.

Gorki, M. 1927 Notes et souvenirs. Paris: Calmann-Lévy.

Keesing, R. 1987 On Anthropology as interpretive quest, Current Anthropology 28 (3) : 356-357.

Mauss, M. 1950 Sociologie et anthropologie. Paris: Presses Universitaires de France.

Meyerson, É. 1931 Le Cheminement de la pensée. Paris: Alcan (3 vol.).

Michotte, A. 1954 La Perception de la causalité. Louvain-Paris: Presses Universitaires de Louvain (Éditions Erasme).

Poirier, J. 1968 L'Étude des cultures matérielles », pp. 560-562, in Ethnologie générale. Paris: Gallimard. Sigaut, F. 1990 Folie, réel et technologie, Techniques et Culture 15: 167-179.

Wallon, H. 1941 L'Évolution psychologique de l'enfant. Paris: Armand Colin.

Weber, L. 1914 Y a-t-il un rythme dans le progrès intellectuel? Bulletin de la Société française de philosophie XIV: 61-140 (séances des 29 janvier et 5 février 1914). 


\section{RÉSUMÉ}

La formule de Mauss. La définition célèbre que donne Mauss de la technique - l'acte traditionnel efficace - peut être traitée comme une formule dans laquelle l'action, la tradition et l'efficacité (la production d'effets physiques) sont les composantes de la technique. Un moyen d'analyser le concept de technique est dès lors d'éliminer successivement chacune de ces trois composantes. Laction traditionnelle sans effet, c'est l'échec ou la panne, mais aussi la simulation (exercices, mime...). L'action efficace, mais dont les effets sont étrangers à la tradition, c'est-àdire paradoxaux, c'est l'innovation, mais aussi la physique amusante et l'illusionnisme de music-hall. Si enfin on a affaire à des effets produits sans action (humaine), il s'agit de phénomènes naturels, réels ou simulés (jeux de hasard, automates). L'existence de ces divers genres d'activités dans de nombreuses sociétés, sinon dans toutes, tend à montrer que le modèle maussien de la technique est présent partout, et que sa validité est donc universelle.

\section{ABSTRACT}

Mauss' formula. One can consider Mauss' famous definition of technology - the efficient traditional act - as a formula in which action, tradition and efficiency (the production of physical effects) are the components of technology. Then, to analyze the concept of technology, one may eliminate in turn each of those three components. The traditional action without effect, i.e. failure or breakdown, but is also simulation (exercise, mimicry...). An efficient action with effects which do not belong to tradition, i.e. paradoxal, is an innovation but is also « fun physics » and music-hall illusionism. Finally, if effects without (human) action happen, we have natural phenomena, real or simulated (gambling, automats). The very existence of these classes of activities in numerous societies, if not all, indicates that Mauss' model of technology is present everywhere and therefore has an universal value.

\section{MOTS CLÉS}

Action, effet, exercice, jeu, mime, physique amusante, prestidigitation, technique, tradition.

\section{KEYWORDS}

Action, effect, exercise, fun physics, music-hall illusionism, technique, tradition.

\section{NOTES ET RÉFÉRENCES DE l'INTRODUCTION}

Bouglé C. 1922 Leçons de sociologie des valeurs. Paris: Armand Colin.

Halbwachs M. 1920 Matière et société, Revue philosophique 90 : 82-122.

Haudricourt A.-G. 1987 La technologie, science humaine. Recherche d'histoire et d'ethnologie des techniques.

Paris: Édition de la Maison des sciences de l'homme.

Mauss M. 1902-1903 Esquisse d’une théorie générale de la magie.

- 1930 Civilisation. Le mot et l'idée. Paris: La renaissance du livre.

— 1936 Les Techniques du corps, Journal de psychologie 32 (3-4). Réédité in Sociologie et anthropologie, Paris: PUF, 1950 : 363-386.

- 2002 (1947) Manuel d'ethnographie. Paris: Payot \& Rivages. 